\title{
Tuning parameters of metal ion implantation within a microfluidic channel
}

\author{
Jae-Woo Choi*ab, Samuel Rosset ${ }^{\mathrm{a}}$, Muhamed Niklaus ${ }^{\mathrm{a}}$, James R. Adleman ${ }^{\mathrm{b}}$, Herbert Shea ${ }^{\mathrm{a}}$, and \\ Demetri Psaltis ${ }^{\mathrm{a}}$ \\ ${ }^{a}$ School of Engineering, Ecole Polytechnique Federale de Lausanne (EPFL), \\ Lausanne, 1015 Switzerland; \\ ${ }^{b}$ Department of Electrical Engineering, California Institute of Technology, \\ Pasadena, CA 91125, United States of America
}

\begin{abstract}
Applying electrical fields is a simple and versatile method to manipulate and reconfigure optofluidic devices. Several methods to apply electric fields using electrodes on polymers or in the context of lab-on-a-chip devices exist. In this paper, we utilize an ion-implanted process to pattern electrodes within a fluidic channel made of polydimethylsiloxane (PDMS). Electrode structuring within the channel is achieved by ion implantation at a $40^{\circ}$ angle with a metal shadow mask. In previous work using the ion-implantation process, we demonstrated two possible applications in the context of lab-on-a-chip applications. Asymmetric particles were aligned through electro-orientation. Colloidal focusing and concentration was possible with negative dielectrophoresis. In this paper, we discuss the different electrode structures that are possible by changing the channel dimensions. A second parameter of ion implantation dosage prevents the shorting of electrodes on the side wall or top wall of the fluidic channel to the bottom. This allows for floating electrodes on the side wall or top wall. These type of electrodes help prevent electrolysis as the liquid is not in direct contact with the voltage source. Possible applications of the different electrode structures that are possible are discussed.
\end{abstract}

Keywords: ion implantation, microfluidics, optofluidics, dielectrophoresis, electro-orientation, polydimethylsiloxane, electric field, bacteria.

\section{INTRODUCTION}

Optofluidics combines microfluidic systems with optical components and methods [1]. The primary benefits of optofluidics are found in integration and reconfigurability. Rather than developing separate chips to do specific tasks in traditional optical systems, in optofluidic systems, we find a number of toolkits that have been developed with the capability to be integrated into a single chip. Another advantage of optofluidics is the ease of reconfigurability. Various mechanisms for the reconfigurability have been realized with the application of external forces. Electrical forces have been utilized to tune a liquid lens, align birefringent material and attract or repel electronic ink [2-4]. Mechanical forces have been utilized to stretch and compress liquid dye laser gratings and to pneumatically pump liquid [5-7]. Optical forces have been utilized to manipulate particles, for example, to form gratings [8-9]. Fluidic replacement has been utilized to demonstrate refractive index tuning and dye lasers [10-14].

Electrodes on polydimethysiloxane (PDMS) have been demonstrated in a variety of methods. One method was by sputtering of metals formed a thin layer of metal on top of the PDMS [15]. Another method is mixing the polymer with conductive material to render the polymer conductive [16]. A disadvantage of these methods is the loss of optical transparency as well as a loss of the polymer properties. On the other hand, metal ion implantation of PDMS modifies the elastic property of polymers mildly while maintaining an optical clear material [17-23]. It has found uses in flexible electronics as well as microactuators, lenses and pressure meters. Moreover, it maintains its conductive properties when stretched to a greater extent than with sputtered gold $[18,22,23]$. It is implanted into the first $50 \mathrm{~nm}$ of the material so the overall polymer properties are not modified as much as if the entire polymer is rendered conductive [17, 19, 20]. Also, it is implanted into the polymer rather than being weakly bonded to the polymer such that a mechanical test with scotch tape will not remove implanted metals but it will remove sputtered metals. Patterning of the ion implantation can be done in a fairly simple manner, through a metal shadow mask or photolithography.

*jae-woo.choi@epfl.ch; phone 4121 693-7828; fax 4121 693-6930; http://lo.epfl.ch

Microfluidics, BioMEMS, and Medical Microsystems VIII, edited by Holger Becker, Wanjun Wang, Proc. of SPIE Vol. 7593, 75930D - (C) 2010 SPIE · CCC code: 1605-7422/10/\$18 - doi: 10.1117/12.842025 
Several methods have been developed to integrate electrodes into microfluidic channels. A substrate with an electrode structuring combined with a microfluidic channel has been very popular [3]. The walls of the microfluidic channel were used as an electrode by etching highly doped silicon [24]. Multi-step optical lithography with etching and metal deposition was used to develop elliptical microrfluidic channels [25]. Vertical electrodes in microfluidic channels were formed through metal deposition and electroplating [26]. Rather than relying on developing electrodes on solid substrates, in this paper, we ion implant into polymers with a microfluidic channel structuring to obtain 3-dimensional electrode patterning.

\section{DESIGN}

\subsection{PDMS fabrication}

Microfluidic channels of various dimensions were fabricated using the replica molding technique. The master mold is produced with UV lithography with SU-8 (Gerstelec) on a silicon wafer. The master mold is subjected to a surface treatment of trimethylsiloxane (TMCS, Sigma-Aldrich) to prevent adhesion to PDMS. PDMS (Dow Corning Sylgard 184 ) is mixed at a 10:1 ratio and poured onto the mold. The entire process is shown in Figure 1.

1. Silicon wafer

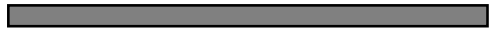

2. Spincoat photoresist

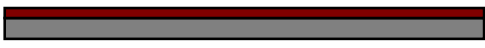

3. UV exposure

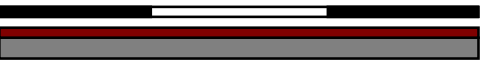

4. Bake

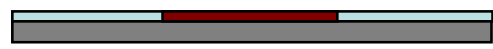

5. Develop

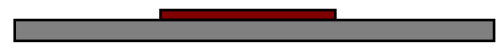

6. Surface treatment with TMCS

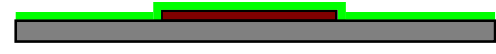

7. Pour PDMS and bake

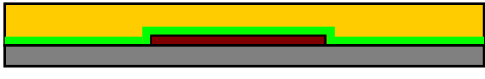

Figure 1. Process flow for fabrication of the master mold and microfluidic channel. 


\subsection{Ion implantation}

A Filtered Cathold Voltage Arc (FCVA) is used to implant gold ions into the PDMS. A steel shadow mask is used to pattern the implanted area. The gold nanoparticles are implanted into the top $50 \mathrm{~nm}$ of the PDMS. The deposition occurs during a pulse when a dense plasma is created between the gold cathode and the trigger electrode (anode). Pulsed at $1 \mathrm{~Hz}$, the filtered ions are accelerated towards the PDMS at a bias of $2.5 \mathrm{keV}$. The ions are doubly charged and the ion energy varies through the pulse. We implant at a $40^{\circ}$ angle to implant ions into the sidewall of the microfluidic channel. The process is repeated twice to coat both sides of the channel. Figure 2 shows the ion implantation process and the angled implantation.
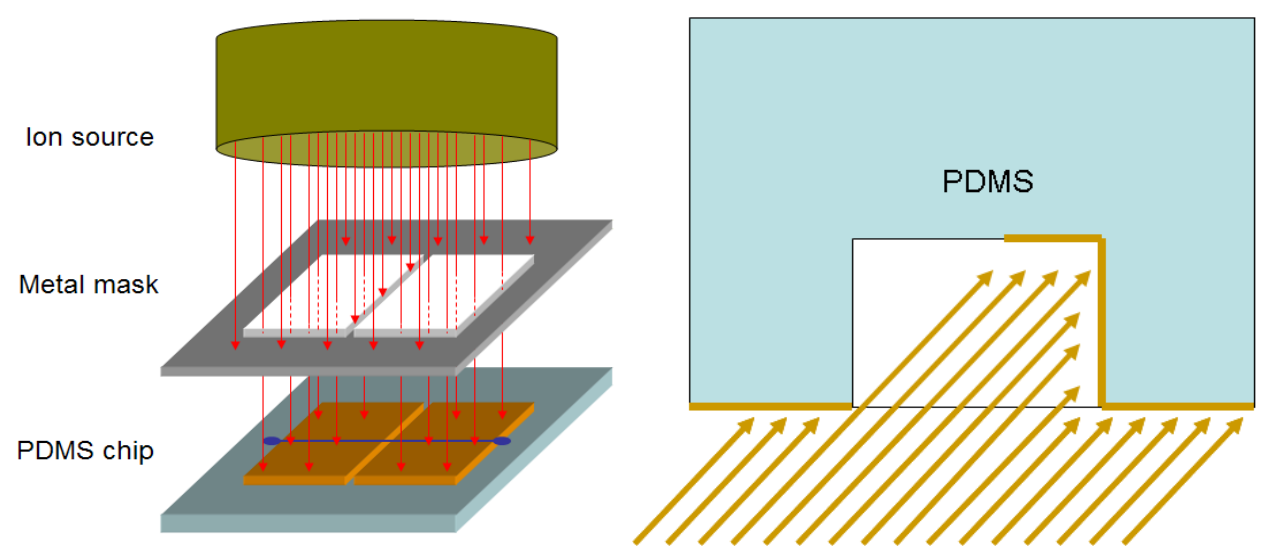

Figure 2. Diagram of ion implantation.

\subsection{Indium tin oxide (ITO) coated glass slide electrode fabrication}

An indium tin oxide (ITO) coated glass slide (Sigma Aldrich) is lithographically pattern to create electrical contact with the gold implanted region as well as add contacts within the fluidic channel. Spin-on-glass (Honeywell) is coated onto specific regions of the ITO coated glass slide to insulate the electrodes for the microfluidic channel from the gold ion implanted region of the PDMS. Inlet and outlet ports are punched into the PDMS with a hole-punching machine and the silicone is given the oxygen plasma treatment for 20 seconds at $50 \mathrm{~W}$. Immediately afterwards, the silicone is bonded to the ITO-coated glass slide. An example of a finished chip design is shown in Figure 3.

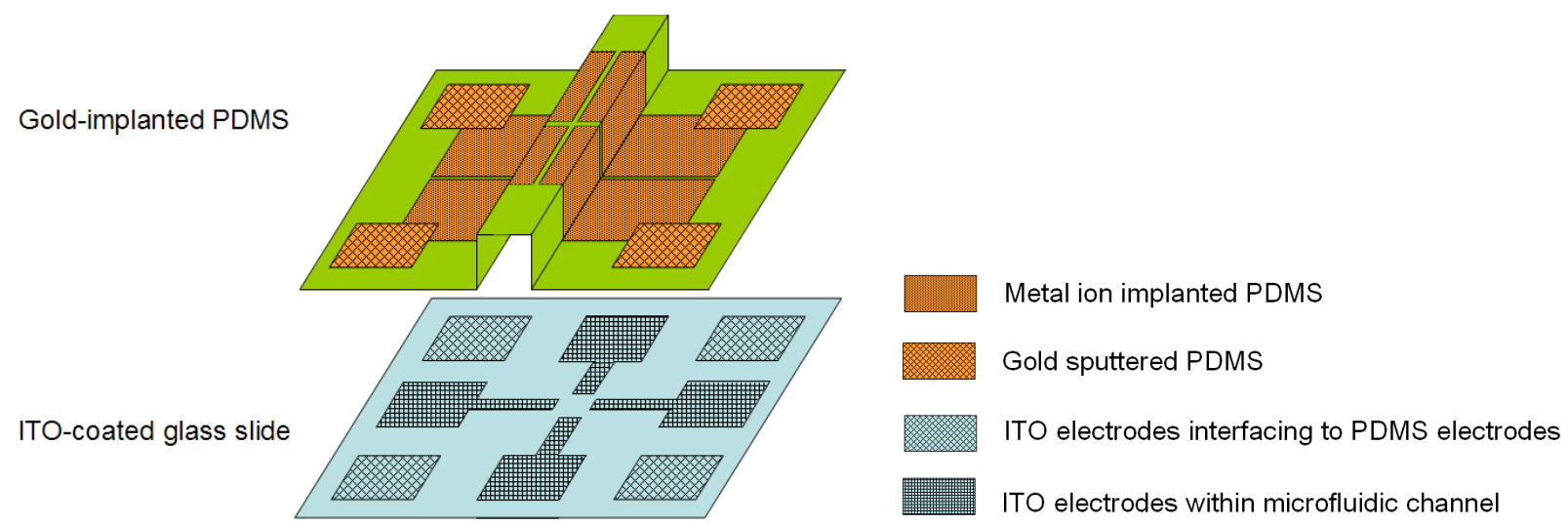

Figure 3. Electrode schematic in PDMS and on ITO coated glass slide. 


\section{TUNING PARAMETERS}

Two primary characteristics in the ion implantation were tuned to achieve different electrode structures. Implantation dosage was modified to prevent connecting the contacts between the bottom, side and top walls of the microfluidic channel. Tuning channel dimensions allows for easily testing the properties of the top wall electrode coating. Changing implantation dosage results in the different possibilities of the ion implantation are shown in Figure 3. The threshold from a single electrode to separate electrodes on each wall of the microfluidic channels occurs at approximately 800 pulses $/ \mathrm{cm}^{2}$.
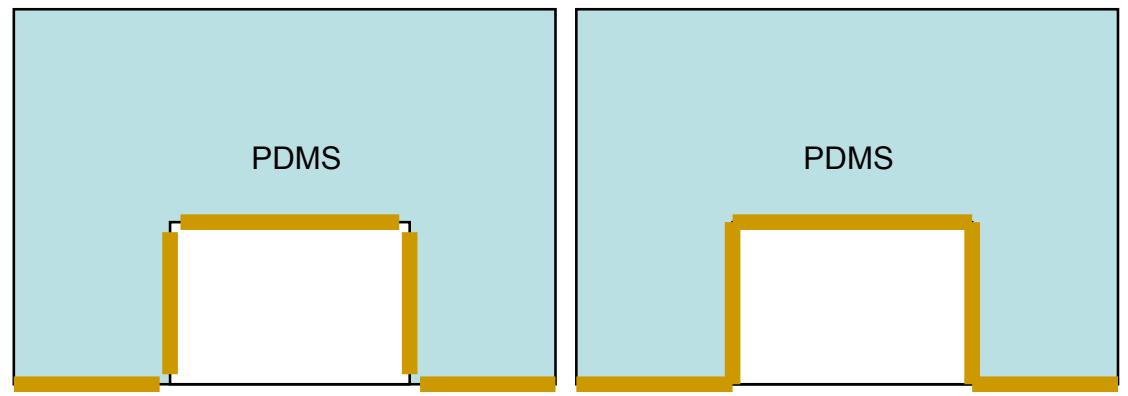

Figure 4. Diagram of different possibilities of ion implantation. Left image shows each wall of the microfluidic channel as separate, unconnected electrodes. Right image shows each wall of the microfluidic channel as a single, connected electrode.

Changing channel dimensions allows for creating different electrode structuring shown in Figure 4 . We utilized a $40^{\circ}$ angle to implant ions at an angle. From this we can calculate the precise conditions to implant electrodes in the different regimes. To describe the uncoated portion of the top wall width for one angled implantation,

$$
\begin{aligned}
& \tan \left(40^{\circ}\right)=\frac{h}{w} \\
& w=h \tan \left(50^{\circ}\right)
\end{aligned}
$$

When $w$ is less than half the total width of the fluidic channel, we are in Regime 1. When $w$ is greater than the total width of the fluidic channel, we are in Regime 3. For values in between, we are in Regime 2. These conclusions were confirmed with fluidic channels of heights of 10 or 70 microns with widths of 30, 50, 70, 80, 90 or 100 microns.
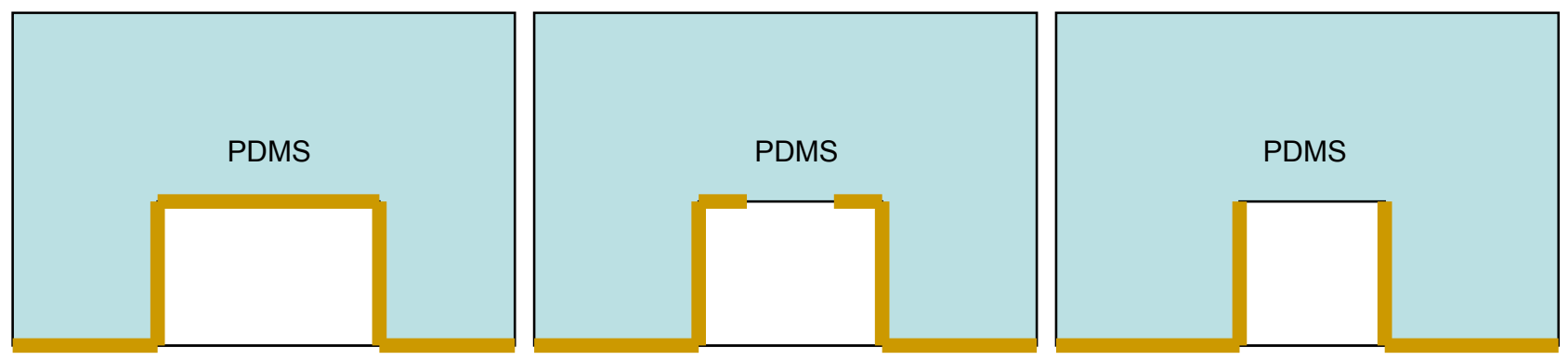

Figure 5. Diagram of different possibilities by tuning microfluidic channel dimensions. Left image shows all walls of the microfluidic channel as a single, connected electrode (Regime 1). Middle image shows a slight coating of the top wall connected to the side and bottom of the chip (Regime 2). Right image shows coating of the side wall only (Regime $3)$. 


\section{BACTERIA MANIPULATION}

Bacteria manipulations were demonstrated using ion-implantation of microfluidic channel in Regime 2 with connected side wall, top wall and bottom electrodes (with the device shown in Figure 3) for electro-orientation and dielectrophoresis [27]. These applications are of particular interest for integration with sensing structures such as the optofluidic microscope.

\subsection{Alignment of bacteria through electro-orientation}

Electro-orientation allows for the alignment of asymmetric particles in liquid to the electric field. We align E coli K12 with $10 \mathrm{~V}_{\mathrm{pp}}$ at $10 \mathrm{MHz}$. Figure 5 shows the images of aligned bacteria. Further details can be found in [27].

(a)
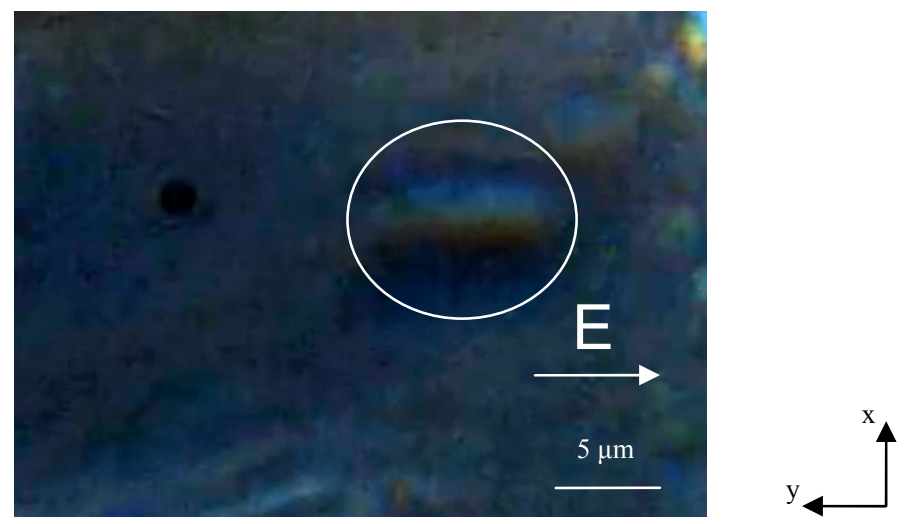

(b)

(c)
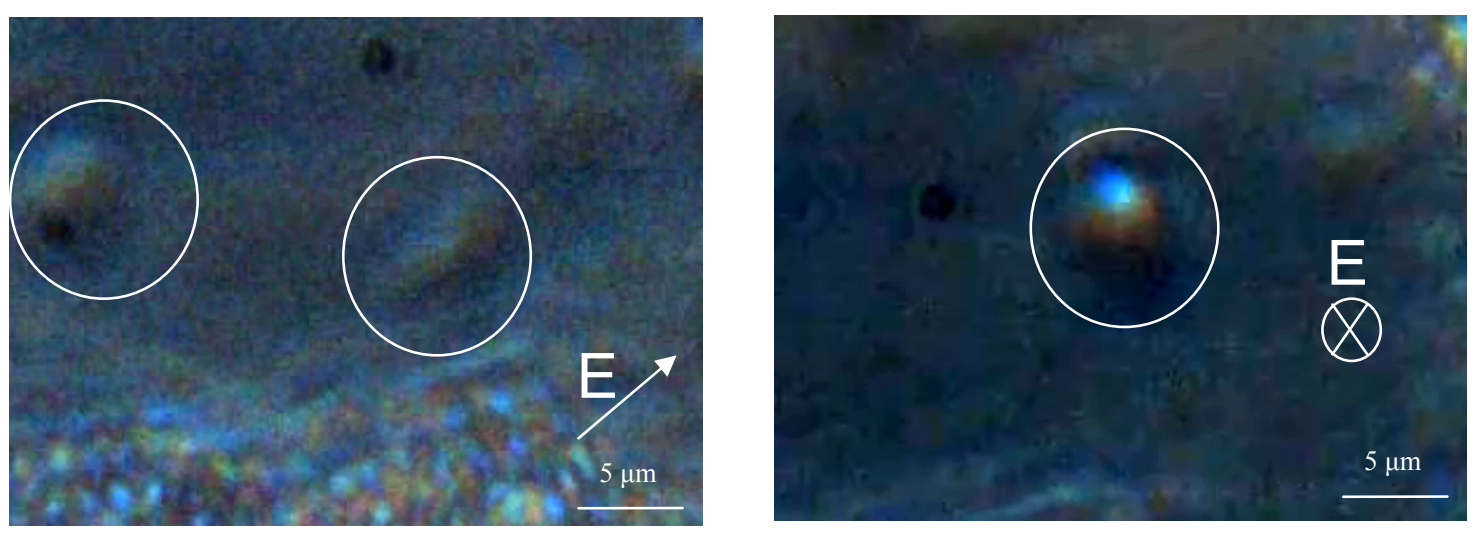

Figure 6. Images of aligned bacteria to the electric field. The direction of the electric field is indicated by the arrows. The aligned bacteria are indicated by the white circles/ellipses. They are aligned in the same direction as to the electric field. 


\subsection{Colloidal focusing through negative dielectrophoresis}

Colloidal focusing was achieved through negative dielectrophoresis. Figure 6 shows the focusing that was achieved by using this force. Further details can be found in [27].

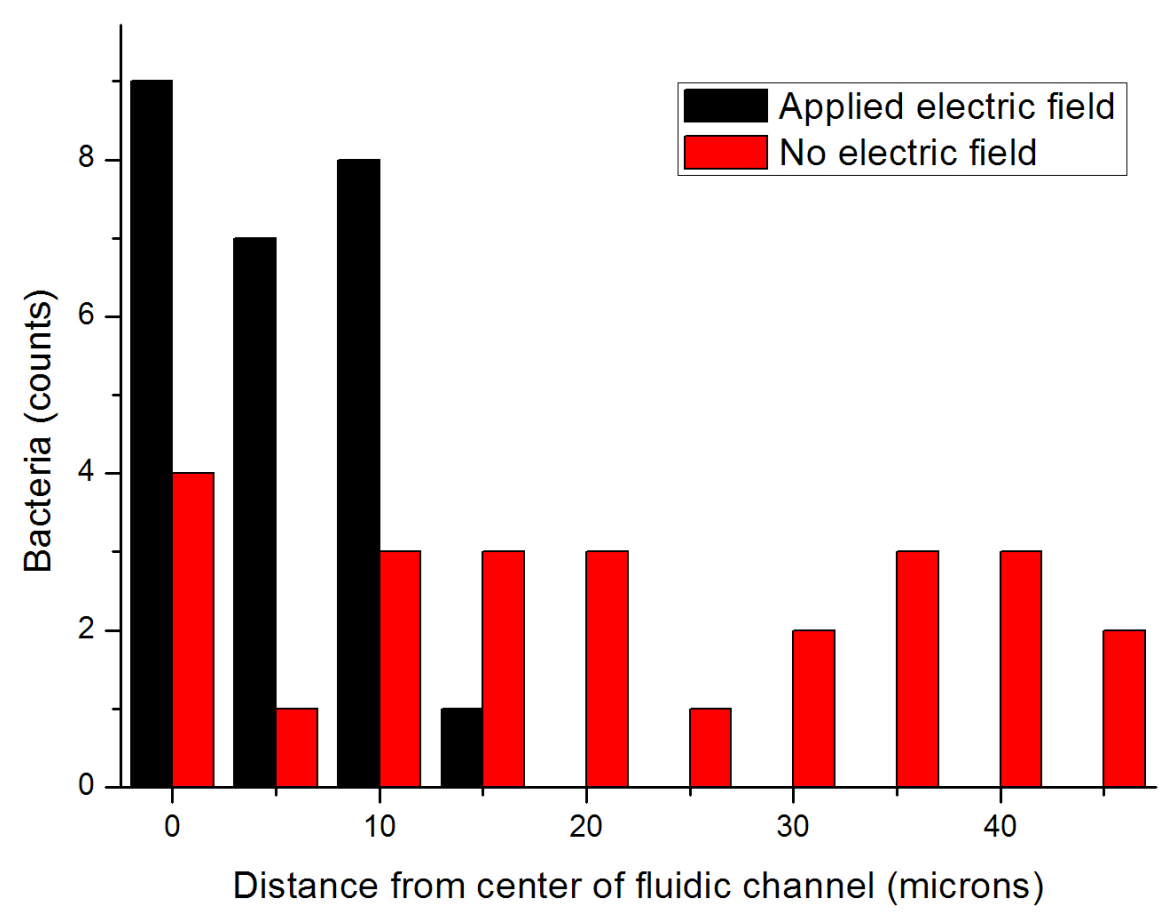

Figure 7. Graph of focused bacteria with applied electric field. The bacteria are concentrated to within 25 microns of the center of the fluidic channel.

\section{CONCLUSION}

Interesting applications are possible with 3-dimensional electrode patterning in microfluidic channels with metal ion implantation of polymers. We demonstrate that different microfluidic channel dimensions allow for distinct regimes where specific electrode patterning is possible. Two possible lab-on-a-chip applications were shown.

\section{ACKNOWLEDGEMENTS}

The authors would like to thank Claude Amendola for cutting the steel metal mask, CMI staff for assistance with SU-8 photolithography process, Rachel Grange and Chia-lung Hsieh for confocal microscope assistance, and Andreas Vasdekis and Wuzhou Song for fluorescence microscope assistance.

\section{REFERENCES}

[1] Psaltis, D., Quake, S. R. and Yang, C., "Developing optofluidic technology through the fusion of microfluidics and optics," Nature 442, 381-386 (2006).

[2] Krupenkin, T., Yang, S. and Mach, P., “Tunable liquid microlens,” Applied Physics Letters 82 (3), 316-318 (2003).

[3] Choi, J.W., Pu, A. and Psaltis, D., "Optical detection of asymmetric bacteria utilizing electro orientation," Optics 
Express 14 (21), 9780-9785 (2006).

[4] Kuiper, S. and Hendriks, B. H. W., "Variable-focus liquid lens for miniature cameras," Applied Physics Letters 85

(7), 1128-1130 (2004).

[5] Li, Z., Zhang, Z., Scherer, A. and Psaltis, D., "Mechanically tunable optofluidic distributed feedback dye laser," Optics Express 14 (22), 10494-10499 (2006).

[6] Unger, M. A., Chou, H. P., Thorsen, T., Scherer, A. and Quake, S. R., "Monolithic microfabricated valves and pumps by multilayer soft lithography," Science 288 (5463), 113-116 (2000).

[7] Li, Z. and Psaltis, D., "Optofluidic dye lasers,” Microfluidics and Nanofluidics 4 (1), 145-158 (2008).

[8] Adleman, J. R., Eggert, H. A., Buse, K. and Psaltis, D., "Holographic grating formation in a colloidal suspension of silver nanoparticles," Optics Letters 31 (4), 447-449 (2006).

[9] Domachuk, P., Cronin-Golomb, M., Eggleton, B. J., Mutzenich, S., Rosengarten, G. and Mitchell, A., “Application of optical trapping to beam manipulation in optofluidics," Optics Express 13 (19), 7265-7275 (2006).

[10] Erickson, D., Rockwood, T., Emery, T., Scherer, A. and Psaltis, D., "Nanofluidic tuning of photonic crystal circuits," Optics Letters 31 (1), 59-61 (2006).

[11] Song, W. Z., Vasdekis, A. E., Li, Z. and Psaltis, D., "Low-order distributed feedback optofluidic dye laser with reduced threshold," Applied Physics Letters 94, 051117 (2009).

[12] Song, W. Z., Vasdekis, A. E., Li, Z. and Psaltis, D. "Optofluidic evanescent dye laser based on a distributed feedback circular grating," Applied Physics Letters 94, 161110 (2009).

[13] Song, W. Z., Zhang, X. M., Liu, A. Q., Yap, P. H. and Hosseini, H., "Refractive index measurement of single living cells using on-chip Fabry-Perot cavity," Applied Physics Letters 89, 203901 (2006).

[14] Tang, S. K. Y., Stan, C. A. and Whitesides, G. M., "Dynamically reconfigurable liquid-core liquid-cladding lens in a microfluidic channel," Lab on a Chip 8, 395-401 (2008).

[15] Lee, K. J., Foster, K. A. and Nuzzo, R. G., "Fabrication of stable metallic pattern embedded in

poly(dimethylsiloxane) and model application in non-planar electronic and lab-on-a-chip device patterning," Advanced Functional Materials 15 (4), 557-566 (2005).

[16] Cong, H. and Pan, T., "Photopatternable conductive PDMS materials for microfabrication," Advanced Functional Materials 18 (13), 1912-1921 (2008).

[17] Rosset, S., Niklaus, M., Dubois, P. and Shea, H., "Large-stroke dielectric elastomer actuators with ion-implanted electrodes," Journal of Microelectromechanical Systems 18 (6), 1300-1308 (2009).

[18] Rosset, S., Niklaus, M., Dubois, P. and Shea, H., "Metal ion implantation for the fabrication of stretchable electrodes on elastomers," Advanced Functional Materials 19 (3), 470-478 (2009).

[19] Dubois, P., Rosset, S., Niklaus, M., Dadras, M. and Shea, H., "Metal ion implanted compliant electrodes in dielectric electroactive polymer (EAP) membrances," Advances in Sciences and Technology 61, 18-25 (2008).

[20] Niklaus, M., Rosset, S., Dadras, M., Dubois, P. and Shea, H., "Microstructure of $5 \mathrm{keV}$ gold-implanted polydimethylsiloxane,” Scripta Materialia 59 (8), 893-896 (2008).

[21] Dubois, P., Rosset, S., Niklaus, M., Dadras, M. and Shea, H., "Voltage control of the resonance frequency of dielectric electroactive polymer (DEAP) membranes," Journal of Microelectromechanical Systems 17 (5), 1072-1081 (2008).

[22] Rosset, S., Niklaus, M., Dubois, P. and Shea, H., "Mechanical characterization of a dielectric elastomer microactuator with ion-implanted electrodes," Sensors and Actuators A: Physical 144 (1), 185-193 (2008).

[23] Dubois, P., Rosset, S., Koster, S., Stauffer, J., Mikhailov, S., Dadras, M., de Rooij, N. F. and Shea, H., "Microactuators based on ion implanted dielectric electroactive polymer (EAP) membranes," Sensors and Actuators A: Physical 130-131, 147-154 (2006).

[24] Ilescu, C., Xu, G. L., Samper, V. and Tay, F. E. H., "Fabrication of a dielectrophoretic chip with 3D silicon electrodes," Journal of Micromechanics and Microengineering 15, 494-500 (2005).

[25] Yu, C., Vykoukal, J., Vykoukal, D. M., Schwartz, J. A., Shi, L. and Gascoyne, P. R. C., "A three-dimensional dielectrophoretic particle focusing channel for microcytometry applications," Journal of Microelectromechanical Systems 14 (3), 480-487 (2005).

[26] Wang, L., Flanagan, L. A., Monuki, E., Jeon, N. L. and Lee, A. P., "Dielectrophoresis switching with vertical sidewall electrodes for microfluidic flow cytometry," Lab on a Chip 7, 1114-1120 (2007).

[27] Choi, J. W., Rosset, S., Niklaus, M., Adleman, J. R., Shea, H. and Psaltis, D., "3-dimensional electrode patterning within a microfluidic channel using a metal ion implantation," Lab on a Chip (2010). doi:10.1039/B917719A. 